\title{
Alterations in the mutagenicity and mutation spectrum induced by benzo[a]pyrene instilled in the lungs of gpt delta mice of various ages
}

Yasunobu Aoki ${ }^{1 *}$, Akiko H. Hashimoto', Yoshiki Sugawara ${ }^{1}$, Kyoko Hiyoshi-Arai $^{1,4}$, Sataro Goto ${ }^{2}$, Kenichi Masumura ${ }^{3}$ and Takehiko Nohmi ${ }^{3}$

\begin{abstract}
Introduction: To examine whether the mutagenic potential of lung exposure to air-borne environmental mutagens is age dependent, we administered $1 \mathrm{mg}$ of benzo[a]pyrene intratracheally to 11- and 24-month old (middle-aged and old, respectively) gpt delta transgenic mice that harbor gpt (guanine phosphoribosyltransferase) genes integrated in the genomic DNA as a target for mutation detection, and then analyzed the benzo[a]pyrene-induced and spontaneous in vivo mutations and mutation spectrum in the lungs.

Results: The mutant frequencies in the lungs of the 11- and 24-month-old control (vehicle-treated) gpt delta mice were $1.14 \pm 0.22 \times 10^{-5}$ and $1.00 \pm 0.20 \times 10^{-5}$, respectively, which are significantly higher than that observed for the control 3-month-old (young) mice $\left(0.59 \pm 0.13 \times 10^{-5}\right)$ in our previous studies, indicating that spontaneous mutation in the lung increases with age. The mutant frequencies in 11- and 24-month-old mice treated with benzo [a] pyrene were 1.5- and 2.3-fold, respectively, that of the age-matched control mice, and 4.3-fold that of the 3-month-old mice in our previous studies. Analysis of mutation spectra showed that both G:C to A:T transitions and G:C to T:A transversions were predominant in the lungs of control mice at all ages. In benzo [a] pyrene-treated mice in our previous studies, G:C to T:A transversions were the predominant type of mutation (55\%) at 3 months. Here we found that their frequency was dramatically reduced to $18 \%$ by 24 months, and the G:C to A:T transitions became the predominant type of mutation in 24-month-old mice (41\% [16\% at CpG sites]).

Conclusions: Our findings suggest that susceptibility to benzo[a]pyrene is highest in young mice and is elevated again in old age. The elevation of G:C to A:T transitions was observed following benzo [a] pyrene administration in the lungs of aged mice, and accelerated cytidine deamination is speculated to contribute to this elevation.
\end{abstract}

Keywords: Aging, Air pollutant, In vivo mutation, Oxidative stress, Transgenic rodent assay

\section{Introduction}

Accumulation of mutations in the genome is considered to be at least in part responsible for the phenomenon of aging. The increase in genomic mutation frequency with age is believed to be a factor in the age-related functional decline of homeostasis and resistance to stressors, which leads to diseases such as cancer [1]. However, it remains to be clarified

\footnotetext{
* Correspondence: ybaoki@nies.go.jp

${ }^{1}$ National Institute for Environmental Studies, Center for Environmental Risk Research, 16-2 Onogawa, 305-8506 Tsukuba, Ibaraki, Japan

Full list of author information is available at the end of the article
}

how vulnerable groups, such as young and old individuals, are susceptible to environmental mutagens and related environmental stressors. For instance, young (3-month-old) rats are more susceptible than adult (11-month-old) rats to acrylamide-induced testicular genotoxicity [2].

Transgenic rodents that harbor exogenous genes integrated in the genomic DNA, as a target for mutation detection, are a useful system for the study of in vivo somatic mutations caused by environmental mutagens. The widely used transgenic lines, Muta mouse, Big Blue rodents, and gpt delta rodents, harbor the Escherichia

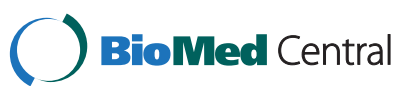


coli genes, lacZ ( $\beta$-galactosidase), lacI (lac repressor), and gpt (guanine phosphoribosyltransferase), respectively [3]. Use of these model animals to evaluate the level of mutations accumulated in aged animals has revealed that the mutation frequency increases spontaneously with age in various organs including liver, spleen, small intestine, kidney, and heart [4-10], but the magnitude of the increase is different among the organs.

From the viewpoint of interactions between the genome and the environment, the lung is a unique organ. In the lung, air-borne environmental mutagens directly contact the pulmonary epithelial cells and induce mutations in the genomic DNA, whereas mutagens reach most organs via the blood circulation system. Therefore, here we chose to use the lung to address how the susceptibility to environmental mutagens differs among age groups. We selected to use benzo[a]pyrene $(\mathrm{B}[\mathrm{a}] \mathrm{P})$, because it is a common air-borne mutagen generated by the burning of fossil fuels. Previously, we reported that $\mathrm{B}[\mathrm{a}] \mathrm{P}$ (0.5-2 mg per animal) induces a linear dosedependent increase in mutation frequency in the lungs of 3-month-old gpt delta mice following a single intratracheal instillation [11]. Here, to determine whether the magnitude of the elevation of in vivo mutant frequency following treatment with an environmental mutagen is age dependent, we examined the mutant frequency and types of mutations in the gpt gene in $\mathrm{B}[\mathrm{a}] \mathrm{P}$-instilled lungs of 11- and 24-month-old gpt delta mice (representative of middle-aged, and old animals, respectively), comparing with those in 3-month-old gpt delta mice (representative of young animals).

\section{Materials and methods}

\section{Treatment of mice}

Male gpt delta mice (9-weeks old; body weight, $\sim 25 \mathrm{~g}$ ), which carry approximately 80 copies of lambda EG10 DNA on each chromosome 17 on a C57BL/6 J background [12], were obtained from Japan SLC (Shizuoka, Japan). The mice were maintained at 24 to $26{ }^{\circ} \mathrm{C}$ with $55 \%$ to $75 \%$ humidity and a 12-h light-dark cycle, and were fed CA-1 diet (Japan Clea Co., Tokyo, Japan) with water ad libitum, in the specific-pathogen-free (SPF) animal facility of the National Institute for Environmental Studies. The animals were anesthetized with $4 \%$ halothane (Hoechst Japan, Tokyo, Japan) in a desiccator until they did not respond to a tactile stimulus. A single dose of B[a]P (1 mg, Wako Pure Chemical Industries, Osaka, Japan) dissolved in $50 \mu \mathrm{L}$ of tricaprylin (Sigma-Aldrich, St. Louis, MO, USA) was intratracheally instilled via a polyethylene tube $[11,13]$. Control mice were treated with $50 \mu \mathrm{L}$ of vehicle (tricaprylin) alone; this dose of $\mathrm{B}[\mathrm{a}] \mathrm{P}$ is within the range $(0.5-2 \mathrm{mg})$ that causes a linear dose-dependent increase in mutant frequency in the lungs of 3-month-old gpt delta mice [11]. The mice were sacrificed 14 days after the administration, and their lungs were removed, frozen in liquid nitrogen, and stored at $-80{ }^{\circ} \mathrm{C}$ until the DNA was isolated. The animal studies were approved by the Animal Care and Use Committee of National Institute for Environmental Studies.

\section{DNA isolation and in vitro packaging of DNA}

High-molecular-weight genomic DNA was extracted from the lungs by using the RecoverEase DNA Isolation Kit (Agilent Technologies, Santa Clara, CA, USA). Lambda EG10 phages containing the gpt gene were recovered from the genomic DNA by using Transpack Packaging Extract (Agilent Technologies).

\section{Mutation assay and DNA sequencing analysis of the gpt gene in 6-TG-resistant colonies}

The gpt mutagenesis assay was performed according to previously described methods [14]. To convert the phage DNA into plasmids, E. coli strain YG6020 expressing Cre recombinase was infected with the rescued phage. The bacteria were then spread onto M9 salts plates containing chloramphenicol $(\mathrm{Cm})$ and 6-thioguanine (6-TG) [14], and incubated for $72 \mathrm{~h}$ at $37^{\circ} \mathrm{C}$ for selection of the colonies harboring a plasmid carrying the chloramphenicol acetyltransferase (cat) gene and a mutated gpt gene. The 6-TG-resistant colonies were streaked onto selection plates for confirmation of the resistant phenotype. The cells were then cultured in LB broth containing $25 \mathrm{mg} / \mathrm{mL} \mathrm{Cm}$ at $37^{\circ} \mathrm{C}$ and collected by centrifugation. The bacterial pellets were stored at $-80{ }^{\circ} \mathrm{C}$ until DNA sequencing analysis was performed. Mutant frequencies for the gpt gene were calculated by dividing the number of colonies growing on $\mathrm{M} 9+\mathrm{Cm}+6-\mathrm{TG}$ agar plates by the number of colonies growing on $\mathrm{M} 9+\mathrm{Cm}$ agar plates. PCR and DNA sequencing analysis of 6-TG-resistant mutants were performed as previously reported [11].

\section{Statistical analysis}

All data are expressed as means \pm SD. Statistical significance was evaluated by using Student's $t$-tests. A statistical analysis of mutational spectra was performed by using the Adams-Skopek test $[15,16]$ and Chi-square test. $P$ values less than 0.05 were considered to be statistically significant.

\section{Results and discussion}

Mutant frequencies in the lungs of B[a]P-treated gpt mice

To examine which age group is most susceptible to a common air-borne environmental mutagen, the mutant frequency and the mutation spectrum in the lungs of 11- and 24-month-old gpt delta mice (representative of middle-aged and old animals, respectively) following treatment with $\mathrm{B}[\mathrm{a}] \mathrm{P}$ and age-matched controls (vehicletreated) were analyzed and compared with our published 
data for 3-month-old mice (representative of young animals) $[11,17,18]$.

The mutant frequencies in the lungs of 11 - and 24-month-old control (vehicle-treated) mice were 1.14 \pm $0.22 \times 10^{-5}$ and $1.00 \pm 0.20 \times 10^{-5}$, respectively. In contrast, the mutant frequency in the lungs of 3-month-old control mice was $0.59 \pm 0.13 \times 10^{-5}$ according to the combined data from our previous reports $[11,17,18]$. Consistent with previously reported observations in various organs such as liver and spleen $[10,19,20]$, these observations indicate that the frequency of spontaneous mutants in the lung increased with age.

A single administration of $1 \mathrm{mg}$ of $\mathrm{B}[\mathrm{a}] \mathrm{P}$ elevated the mutant frequency in 11 - and 24-month-old mice to $1.68 \pm 0.19 \times 10^{-5}$ and $2.25 \pm 0.54 \times 10^{-5}$, respectively, which was 1.5 - and 2.3-fold the mutant frequency in the respective age-matched control mice (Table 1 ). We previously reported that $\mathrm{B}[\mathrm{a}] \mathrm{P}$ instillation to the lungs of 3-month-old mice elevated the mutant frequency to $2.52 \pm 0.33 \times 10^{-5}$ [11] which was 4.3 -fold the frequency observed in the 3-month-old control mice in our previous studies $[11,17,18]$.

Our observations indicate that the order of the age groups in terms of highest to lowest fold of increase in mutant frequency in the lungs following instillation of $\mathrm{B}[\mathrm{a}] \mathrm{P}$ was 3-, 24-, and 11-month-old mice. These results suggest that young mice are the age-group most susceptible to $\mathrm{B}[\mathrm{a}] \mathrm{P}$, which may be explained age-related changes of the mutant frequency by their relatively high level of DNA replicating activity and cell turnover rate [21] and/or perhaps higher levels of metabolic activation of $\mathrm{B}[\mathrm{a}] \mathrm{P}$, as well as DNA repair activity, which promote the formation of $\mathrm{B}[\mathrm{a}] \mathrm{P}-\mathrm{DNA}$ adducts, and that the susceptibility is elevated again in old age by several possible mechanisms, as discussed later. Age-dependent alteration in the metabolic activation of $\mathrm{B}[\mathrm{a}] \mathrm{P}$ has not been well-documented in the lung, but the activity of cytochrome 1A, a mono-oxygenase that catalyzes the metabolic activation of $\mathrm{B}[\mathrm{a}] \mathrm{P}$, has been suggested to decline with age in the rat liver [22].

\section{Characteristics of the gpt mutation spectrum}

To analyze the age-dependent alterations in the mutation spectra of B[a]P-instilled and control lungs, we sequenced gpt mutants recovered from the lungs as shown in Table 2. We observed a significant difference between the mutation spectrum of the 24-month-old control mice studied here and that of the 3-month-old control mice we studied previously $[11,17,18](P<0.05$, Adams-Skopec test). In our published data from 3-month-old control mice, the most predominant type of base substitution was $G: C$ to A:T transitions ( $45 \%$ of total mutants), and a half of these transitions were induced at $\mathrm{CpG}$ sites (18\% of total mutants), while G:C to T:A (21 \%) and G:C to C:G (16 \%) transversions were also major base substitutions. Here, were found that the percentages of $\mathrm{G}: \mathrm{C}$ to $\mathrm{C}: \mathrm{G}$ transversions among total mutants were less in 11- and 24month-old control mice than in 3-month-old control mice, but $\mathrm{G}: \mathrm{C}$ to $\mathrm{A}: \mathrm{T}$ transitions and $\mathrm{G}: \mathrm{C}$ to $\mathrm{T}: \mathrm{A}$ transversions were still predominant $(35 \%$ and $14 \%$ of total mutants, respectively) in 24-month-old control mice (Table 2). In contrast, increased proportions of A:T to T:A transversions, A:T to $\mathrm{C}: \mathrm{G}$ transversions, and base deletions were observed in the lungs of 24-month-old control mice compared with 3-month-old control mice. An increase in base substitutions at A:T has previously been reported in the intestine and spleen of 32-month-old lac Z plasmid transgenic mice, and DNA polymerase $\eta$ was speculated to act as an A:T mutator in the spleen of aged animals [23]. Taken together, these results suggest that an increase in point mutations at $\mathrm{A}: \mathrm{T}$ may be a common phenomenon in proliferative aged tissues.

We observed that there was a significant difference between the mutation spectra for both 3- and 11-month-old $\mathrm{B}$ [a]P-instilled mice $(P<0.05$, Adams-Skopec test $)$, and 3 - and 24-month-old B[a]P-instilled mice $(P<0.01$, Adams-Skopec test). In $\mathrm{B}[\mathrm{a}] \mathrm{P}$-instilled groups, we reported previously that $\mathrm{G}: \mathrm{C}$ to $\mathrm{T}: \mathrm{A}$ transversions, a major base substitution induced by $\mathrm{B}[\mathrm{a}] \mathrm{P}$ administration, were the predominant type of mutation (55\%) in the lungs of 3-month-old mice in our previous study [11], but surprisingly in the aged mice, the percentage of these mutations was dramatically lowered to be $24 \%$ and $18 \%$ in 11 - and 24-month-old mice, respectively (Table 2 ). In contrast, the percentage of $\mathrm{G}: \mathrm{C}$ to $\mathrm{A}: \mathrm{T}$ transitions was observed to increase in $\mathrm{B}[\mathrm{a}] \mathrm{P}$-instilled lungs age-dependently, and they became the predominant type of mutation in 24-monthold mice (41 \% [16 \% at CpG sites]). As observed for control lungs, the percentage of $\mathrm{G}: \mathrm{C}$ to $\mathrm{C}: \mathrm{G}$ transversions in $\mathrm{B}[\mathrm{a}] \mathrm{P}$-instilled lungs decreased with age.

The positions of spontaneous and $\mathrm{B}[\mathrm{a}] \mathrm{P}$-induced $g p t$ mutations are listed in Table 3. Among the mutated sequences isolated from the control mice, $6 \mathrm{gpt}$ mutations (G:C to A:T transitions) at nucleotides $64,86,115$, and 406 in 11-month-old mice, at nucleotide 110 in 4 and 24-month-old mice, and at nucleotide 417 in 24month-old mice, were each observed in three or more mice. These positions therefore are potential hotspots, while G:C to A:T transitions at nucleotides 64 and 110 were reported to be sites of spontaneous mutation in $g p t$ delta mice [24]. The G:C to A:T transitions at nucleotides 64 and 110 and the $G: C$ to $C: G$ transversion at nucleotide 340 were also hotspots in 11-month-old $\mathrm{B}[\mathrm{a}] \mathrm{P}$-instilled mice. Regarding G:C to T:A transversions, we previously observed that nucleotides 115, 140, 143, 189 , and 413 were hotspots for $\mathrm{G}: \mathrm{C}$ to $\mathrm{T}: \mathrm{A}$ transversions in $\mathrm{B}[\mathrm{a}] \mathrm{P}$-instilled lungs of 3-month-old gpt delta mice [11], but these nucleotides were not hotspots for this 
Table 1 Mutant frequencies in B[a]P-instilled and control lung of gpt delta mice

\begin{tabular}{|c|c|c|c|c|c|c|}
\hline \multirow{2}{*}{$\begin{array}{l}\mathrm{B}[\mathrm{a}] \mathrm{P} \\
(\mathrm{mg}) \\
\end{array}$} & \multirow{2}{*}{$\begin{array}{l}\text { Time } \\
\text { (months) }\end{array}$} & \multirow[t]{2}{*}{ ID of animals } & \multicolumn{2}{|c|}{ Number of colonies } & \multirow{2}{*}{$\begin{array}{l}\text { Mutant frequency } \\
\left(\times 10^{-5}\right)\end{array}$} & \multirow{2}{*}{$\begin{array}{l}\text { Average mutant } \\
\text { frequency } \pm S D \\
\left(\times 10^{-5}\right)\end{array}$} \\
\hline & & & Mutant & Total & & \\
\hline \multirow[t]{20}{*}{0} & 3 & $1^{\mathrm{a}}$ & 5 & 800,800 & 0.62 & \\
\hline & & $2^{\mathrm{a}}$ & 5 & $1,113,600$ & 0.45 & \\
\hline & & $3^{\mathrm{a}}$ & 5 & 702,400 & 0.71 & \\
\hline & & $4^{b}$ & 3 & 441,600 & 0.68 & \\
\hline & & $5^{\mathrm{b}}$ & 3 & 643,200 & 0.47 & \\
\hline & & $6^{\mathrm{b}}$ & 3 & 828,000 & 0.36 & \\
\hline & & $7^{c}$ & 7 & $1,016,000$ & 0.69 & \\
\hline & & $8^{c}$ & 6 & 836,800 & 0.72 & \\
\hline & & $9^{c}$ & 3 & 524,200 & 0.57 & \\
\hline & & Total & 40 & $6,906,600$ & & $0.59 \pm 0.13$ \\
\hline & 11 & 1 & 3 & 311,000 & 0.96 & \\
\hline & & 2 & 5 & 530,000 & 0.94 & \\
\hline & & 3 & 30 & $2,389,000$ & 1.26 & \\
\hline & & 4 & 28 & $1,915,000$ & 1.46 & \\
\hline & & 5 & 34 & $3,117,000$ & 1.09 & \\
\hline & & Total & 100 & $8,262,000$ & & $1.14 \pm 0.22^{+\dagger}$ \\
\hline & 24 & 1 & 12 & $1,408,000$ & 0.85 & \\
\hline & & 2 & 18 & $1,464,000$ & 1.23 & \\
\hline & & 3 & 14 & $1,548,000$ & 0.90 & \\
\hline & & Total & 44 & $4,420,000$ & & $1.00 \pm 0.20^{\dagger}$ \\
\hline \multirow[t]{14}{*}{1} & 3 & $1^{a}$ & 11 & 499,200 & 2.20 & \\
\hline & & $2^{a}$ & 14 & 556,800 & 2.51 & \\
\hline & & $3^{a}$ & 35 & $1,225,600$ & 2.86 & \\
\hline & & Total & 60 & $2,281,600$ & & $2.52 \pm 0.33^{* *}$ \\
\hline & 11 & 1 & 11 & 750,000 & 1.47 & \\
\hline & & 2 & 13 & 700,000 & 1.86 & \\
\hline & & 3 & 14 & 738,000 & 1.90 & \\
\hline & & 4 & 17 & $1,061,000$ & 1.60 & \\
\hline & & 5 & 27 & $1,728,000$ & 1.56 & \\
\hline & & Total & 82 & $4,977,000$ & & $1.68 \pm 0.19^{*,+\dagger}$ \\
\hline & 24 & 1 & 13 & 456,000 & 2.85 & \\
\hline & & 2 & 36 & $1,996,000$ & 1.80 & \\
\hline & & 3 & 17 & 809,000 & 2.10 & \\
\hline & & Total & 66 & $3,261,000$ & & $2.25 \pm 0.54^{*}$ \\
\hline
\end{tabular}

${ }^{*} P<0.01,{ }^{* *} P<0.001$ for comparison between $\mathrm{B}[a] \mathrm{P}$-instilled mice and age-matched control mice

${ }^{\dagger} P<0.01,{ }^{\dagger \dagger} P<0.001$ for comparison to equivalently treated 3-month-old mice

$\mathrm{a}, \mathrm{b}$ and ${ }^{\mathrm{C}}$ Data from our previous studies $([11,17,18]$, respectively)

base substitution in 11- and 24-month-old mice; rather the hotspots were nucleotides 402 and 406 in B[a]Pinstilled lungs of 11-month-old mice, but there was no hotspot in 24-month-old mice.

Our results showed that the predominant type of mutation in the lungs of the vehicle control gpt delta mice was G:C to A:T transitions in all age groups; these transitions have also been shown to be the predominant type of mutation in liver and other organs in both newborn and 23-month-old lacZ-transgenic mouse [19]. G:C to A:T transitions are recognized to be more frequently induced on CpG sites, in which cytosines tend to be 
Table 2 Classification of gpt mutations from the lung of B[a]P-instilled and control mice

\begin{tabular}{|c|c|c|c|c|c|c|c|c|c|c|c|c|c|c|c|c|}
\hline \multirow{3}{*}{$\begin{array}{l}\text { Type of mutation in the } \\
\text { gpt gene }\end{array}$} & \multirow{2}{*}{\multicolumn{2}{|c|}{$\begin{array}{l}\text { Control } \\
\text { All ages }\end{array}$}} & \multirow{2}{*}{\multicolumn{2}{|c|}{$\begin{array}{l}\text { B[a]P } \\
\text { All ages }\end{array}$}} & \multicolumn{6}{|c|}{ Control (months) } & \multicolumn{6}{|c|}{$\mathrm{B}[\mathrm{a}] \mathrm{P}$ (months) } \\
\hline & & & & & \multicolumn{2}{|c|}{$3^{\mathrm{a}}$} & \multicolumn{2}{|l|}{11} & \multicolumn{2}{|l|}{24} & \multicolumn{2}{|c|}{$3^{b}$} & \multicolumn{2}{|l|}{11} & \multicolumn{2}{|l|}{24} \\
\hline & Number & $\%$ & Number & $\%$ & Number & $\%$ & Number & $\%$ & Number & $\%$ & Number & $\%$ & Number & $\%$ & Number & $\%$ \\
\hline \multicolumn{17}{|l|}{ Base substitution } \\
\hline \multicolumn{17}{|l|}{ Transition } \\
\hline $\mathrm{G}: \mathrm{C} \rightarrow \mathrm{A}: \mathrm{T}$ & 68 & 39 & 44 & 26 & 17 & 45 & 36 & 39 & 15 & 35 & 4 & 10 & 20 & 25 & 20 & 41 \\
\hline (at CpG site) & (28) & (16) & (20) & (12) & (7) & (18) & (14) & (15) & (7) & (16) & (1) & (2) & (11) & (13) & (8) & (16) \\
\hline $\mathrm{A}: \mathrm{T} \rightarrow \mathrm{G}: \mathrm{C}$ & 14 & 8 & 1 & 1 & 3 & 8 & 10 & 11 & 1 & 2 & 0 & 0 & 0 & 0 & 1 & 2 \\
\hline \multicolumn{17}{|l|}{ Transversion } \\
\hline $\mathrm{G}: \mathrm{C} \rightarrow \mathrm{T}: \mathrm{A}$ & 29 & 17 & 51 & 30 & 8 & 21 & 15 & 16 & 6 & 14 & 23 & 55 & 19 & 24 & 9 & 18 \\
\hline (at CpG site) & (6) & (3) & (33) & (19) & (3) & (8) & (3) & (3) & (1) & (2) & (19) & (45) & (8) & (10) & (6) & (12) \\
\hline $\mathrm{G}: \mathrm{C} \rightarrow \mathrm{C}: \mathrm{G}$ & 12 & 7 & 22 & 13 & 6 & 16 & 4 & 4 & 2 & 5 & 7 & 17 & 12 & 15 & 3 & 6 \\
\hline $\mathrm{A}: \mathrm{T} \rightarrow \mathrm{T}: \mathrm{A}$ & 12 & 7 & 7 & 4 & 1 & 3 & 6 & 7 & 5 & 12 & 0 & 0 & 4 & 5 & 3 & 6 \\
\hline $\mathrm{A}: \mathrm{T} \rightarrow \mathrm{C}: \mathrm{G}$ & 7 & 4 & 3 & 2 & 0 & 0 & 4 & 4 & 3 & 7 & 0 & 0 & 0 & 0 & 3 & 6 \\
\hline \multicolumn{17}{|l|}{ Deletion } \\
\hline-1 & 18 & 10 & 22 & 13 & 3 & 8 & 9 & 10 & 6 & 14 & 4 & 10 & 14 & 18 & 4 & 8 \\
\hline$>2$ & 6 & 3 & 5 & 3 & 0 & 0 & 2 & 2 & 4 & 9 & 0 & 0 & 2 & 3 & 3 & 6 \\
\hline Insertion & 6 & 3 & 11 & 6 & 0 & 0 & 5 & 5 & 1 & 2 & 2 & 5 & 6 & 8 & 3 & 6 \\
\hline Other & 1 & 1 & 4 & 2 & 0 & 0 & 1 & 1 & 0 & 0 & 2 & 5 & 2 & 3 & 0 & 0 \\
\hline Total & 173 & 100 & 170 & 100 & 38 & 100 & 92 & 100 & 43 & 100 & 42 & 100 & 79 & 100 & 49 & 100 \\
\hline
\end{tabular}

${ }^{\mathrm{a}}$ Combined data of our previous studies $[11,17,18]$

Our previous data [11] 
Table 3 DNA sequence analysis of gpt mutations obtained from the lung of B[a]P-instilled and control mice

\begin{tabular}{|c|c|c|c|c|c|c|c|c|c|c|c|c|c|c|}
\hline \multirow{4}{*}{$\begin{array}{l}\text { Type of } \\
\text { mutation }\end{array}$} & \multirow[t]{4}{*}{ Nucleo-tide \# } & \multicolumn{2}{|c|}{ Sequence } & \multicolumn{2}{|c|}{ Change } & \multicolumn{3}{|c|}{ Amino acid change } & \multicolumn{6}{|c|}{ Number } \\
\hline & & & & & & & & & \multirow{2}{*}{\multicolumn{3}{|c|}{$\begin{array}{l}\text { Control } \\
\text { Months }\end{array}$}} & \multirow{2}{*}{\multicolumn{3}{|c|}{$\begin{array}{l}\mathrm{B}[\mathrm{a}] \mathrm{P} \\
\text { Months }\end{array}$}} \\
\hline & & & & & & & & & & & & & & \\
\hline & & & & & & & & & $3^{d}$ & 11 & 24 & $3^{\mathrm{e}}$ & 11 & 24 \\
\hline \multicolumn{15}{|c|}{ Base substitution } \\
\hline \multicolumn{15}{|l|}{ Transition } \\
\hline \multirow[t]{24}{*}{$\mathrm{G}: \mathrm{C} \rightarrow \mathrm{A}: \mathrm{T}$} & 26 & $\mathrm{tGg}$ & $\rightarrow$ & $\mathrm{tAg}$ & & Trp & $\rightarrow$ & Stop & & & 1 & & $2^{\mathrm{a}}$ & 1 \\
\hline & 27 & $\operatorname{tg} G$ & $\rightarrow$ & $\operatorname{tg} A$ & & Trp & $\rightarrow$ & Stop & & & 1 & & 1 & \\
\hline & 37 & Cag & $\rightarrow$ & Tag & & Gln & $\rightarrow$ & Stop & & & 1 & & 2 & 1 \\
\hline & 58 & Gca & $\rightarrow$ & Aca & CpG & Ala & $\rightarrow$ & Thr & & & 1 & & & \\
\hline & 64 & Cga & $\rightarrow$ & Tga & $\mathrm{CpG}$ & $\operatorname{Arg}$ & $\rightarrow$ & Stop & 1 & $5^{b}$ & 1 & & $5^{b}$ & 1 \\
\hline & 86 & $\mathrm{tGg}$ & $\rightarrow$ & tAg & & Trp & $\rightarrow$ & Stop & & $3^{b}$ & & 1 & & \\
\hline & 87 & $\operatorname{tg} G$ & $\rightarrow$ & $\operatorname{tg} A$ & & Trp & $\rightarrow$ & Stop & & $2^{\mathrm{a}}$ & & & & \\
\hline & 92 & gGc & $\rightarrow$ & $g A c$ & & Gly & $\rightarrow$ & Asp & & $2^{\mathrm{a}}$ & & & & \\
\hline & 110 & $c G t$ & $\rightarrow$ & cAt & $\mathrm{CpG}$ & $\operatorname{Arg}$ & $\rightarrow$ & His & $5^{c}$ & 4 & $3^{b}$ & & $4^{b}$ & $6^{a}$ \\
\hline & 115 & Ggt & $\rightarrow$ & Agt & $\mathrm{CpG}$ & Gly & $\rightarrow$ & Ser & 1 & $5^{b}$ & 2 & 1 & $2^{a}$ & 1 \\
\hline & 116 & gGt & $\rightarrow$ & gAt & & Gly & $\rightarrow$ & Asp & 2 & $2^{\mathrm{a}}$ & & & & 1 \\
\hline & 128 & gGt & $\rightarrow$ & gAt & & Gly & $\rightarrow$ & Asp & 1 & 2 & & & & 2 \\
\hline & 176 & $\mathrm{tGt}$ & $\rightarrow$ & tAt & & Cys & $\rightarrow$ & Tyr & & & & & & 1 \\
\hline & 262 & Gat & $\rightarrow$ & Aat & & Asp & $\rightarrow$ & Asn & 1 & & & & & \\
\hline & 274 & Gat & $\rightarrow$ & Aat & & Asp & $\rightarrow$ & Asn & 1 & & 1 & & 1 & \\
\hline & 281 & gGt & $\rightarrow$ & gAt & & Gly & $\rightarrow$ & Asp & & & & & & 1 \\
\hline & 284 & gGt & $\rightarrow$ & gAt & & Gly & $\rightarrow$ & Asp & & & & & & 1 \\
\hline & 367 & Gat & $\rightarrow$ & Aat & & Asp & $\rightarrow$ & Asn & & & & & 1 & \\
\hline & 401 & $\mathrm{tGg}$ & $\rightarrow$ & $\mathrm{tAg}$ & & Trp & $\rightarrow$ & Stop & $2^{\mathrm{a}}$ & $4^{\mathrm{a}}$ & & & & 1 \\
\hline & 402 & $\operatorname{tg} G$ & $\rightarrow$ & $\operatorname{tg} A$ & & Trp & $\rightarrow$ & Stop & & & & 1 & & 1 \\
\hline & 406 & Gaa & $\rightarrow$ & Aaa & & Glu & $\rightarrow$ & Lys & & $3^{b}$ & & & & \\
\hline & 416 & $\mathrm{tGg}$ & $\rightarrow$ & $\mathrm{tAg}$ & & Trp & $\rightarrow$ & Stop & & 1 & & & & \\
\hline & 417 & $\operatorname{tg} G$ & $\rightarrow$ & $\operatorname{tg} A$ & & Trp & $\rightarrow$ & Stop & 1 & 1 & $4^{b}$ & & & \\
\hline & 418 & Gat & $\rightarrow$ & Aat & & Asp & $\rightarrow$ & Asn & $2^{\mathrm{a}}$ & $2^{\mathrm{a}}$ & & 1 & $2^{\mathrm{a}}$ & $2^{a}$ \\
\hline \multirow[t]{5}{*}{$\mathrm{A}: \mathrm{T} \rightarrow \mathrm{G}: \mathrm{C}$} & 25 & Tgg & $\rightarrow$ & Cgg & & Trp & $\rightarrow$ & Arg & 1 & & & & & \\
\hline & 41 & aTc & $\rightarrow$ & $\mathrm{aCc}$ & & Ile & $\rightarrow$ & Thr & 1 & & & & & \\
\hline & 56 & cTc & $\rightarrow$ & $c C c$ & & Leu & $\rightarrow$ & Pro & 1 & $8^{a}$ & 1 & & & \\
\hline & 149 & cTg & $\rightarrow$ & $\mathrm{cCg}$ & & Leu & $\rightarrow$ & Pro & & 1 & & & & 1 \\
\hline & 415 & Tgg & $\rightarrow$ & Cgg & & Trp & $\rightarrow$ & Arg & & 1 & & & & \\
\hline \multicolumn{15}{|l|}{ Transversion } \\
\hline \multirow[t]{9}{*}{$\mathrm{G}: \mathrm{C} \rightarrow \mathrm{T}: \mathrm{A}$} & 3 & atG & $\rightarrow$ & atT & & Met & $\rightarrow$ & lle & & 1 & & & & \\
\hline & 7 & Gaa & $\rightarrow$ & Taа & CpG & Glu & $\rightarrow$ & Stop & & 1 & & & & \\
\hline & 15 & $\mathrm{taC}$ & $\rightarrow$ & taA & & Tyr & $\rightarrow$ & Stop & & 1 & & & & \\
\hline & 26 & $\mathrm{tGg}$ & $\rightarrow$ & $\mathrm{tTg}$ & & Trp & $\rightarrow$ & Leu & & & & & & 1 \\
\hline & 37 & Cag & $\rightarrow$ & Aag & & Gln & $\rightarrow$ & Lys & & & 1 & & & \\
\hline & 79 & Gaa & $\rightarrow$ & Taа & & Glu & $\rightarrow$ & Stop & & & & & 1 & \\
\hline & 92 & gGc & $\rightarrow$ & gTc & & Gly & $\rightarrow$ & Val & & & & & 1 & \\
\hline & 101 & $\mathrm{gCc}$ & $\rightarrow$ & gAc & & Ala & $\rightarrow$ & Asp & & & & & 1 & \\
\hline & 108 & $\mathrm{agC}$ & $\rightarrow$ & $\operatorname{agA}$ & & Ser & $\rightarrow$ & Arg & & & & 2 & & \\
\hline
\end{tabular}


Table 3 DNA sequence analysis of gpt mutations obtained from the lung of B[a]P-instilled and control mice (Continued)

\begin{tabular}{|c|c|c|c|c|c|c|c|c|c|c|c|c|c|c|}
\hline & 110 & $c G t$ & $\rightarrow$ & $c T t$ & CpG & $\operatorname{Arg}$ & $\rightarrow$ & Leu & & & & & & 1 \\
\hline & 115 & Ggt & $\rightarrow$ & Tgt & CpG & Gly & $\rightarrow$ & Cys & & & & 2 & 1 & $2^{a}$ \\
\hline & 116 & gGt & $\rightarrow$ & $\mathrm{gTt}$ & & Gly & $\rightarrow$ & Val & & $2^{a}$ & & & & 1 \\
\hline & 140 & $\mathrm{gCg}$ & $\rightarrow$ & $\mathrm{gAg}$ & CpG & Ala & $\rightarrow$ & Glu & 1 & & & 1 & $3^{a}$ & 1 \\
\hline & 143 & $\mathrm{cGt}$ & $\rightarrow$ & $c T t$ & CpG & Arg & $\rightarrow$ & Leu & & & & $8^{a}$ & 1 & \\
\hline & 145 & Gaa & $\rightarrow$ & Taa & & Glu & $\rightarrow$ & Stop & 1 & 1 & 1 & & & \\
\hline & 185 & $\mathrm{aGc}$ & $\rightarrow$ & aTc & & Ser & $\rightarrow$ & lle & 1 & & & & & 1 \\
\hline & 186 & $\operatorname{agC}$ & $\rightarrow$ & $\operatorname{agA}$ & & Ser & $\rightarrow$ & Arg & 1 & & & & & \\
\hline & 189 & $\mathrm{taC}$ & $\rightarrow$ & taA & $\mathrm{CpG}$ & Tyr & $\rightarrow$ & Stop & & & 1 & $3^{a}$ & 1 & \\
\hline & 208 & Gag & $\rightarrow$ & Tag & CpG & Glu & $\rightarrow$ & Stop & & & & 3 & 1 & \\
\hline & 244 & Gaa & $\rightarrow$ & Taa & CpG & Glu & $\rightarrow$ & Stop & 1 & $2^{a}$ & & 2 & 1 & 1 \\
\hline & 262 & Gat & $\rightarrow$ & Tat & & Asp & $\rightarrow$ & Tyr & & & 1 & & & \\
\hline & 281 & gGt & $\rightarrow$ & $\mathrm{gTt}$ & & Gly & $\rightarrow$ & Val & & & & & 1 & \\
\hline & 304 & Gaa & $\rightarrow$ & Taa & & Glu & $\rightarrow$ & Stop & 1 & 1 & 1 & & & \\
\hline & 320 & $\mathrm{gCg}$ & $\rightarrow$ & gAg & CpG & Ala & $\rightarrow$ & Glu & 1 & & & & & 1 \\
\hline & 401 & $\mathrm{tGg}$ & $\rightarrow$ & $\mathrm{tTg}$ & & Trp & $\rightarrow$ & Leu & & $2^{a}$ & & & & \\
\hline & 402 & $\operatorname{tg} G$ & $\rightarrow$ & $\operatorname{tg} T$ & & Trp & $\rightarrow$ & Cys & & 1 & & 1 & $4^{b}$ & \\
\hline & 406 & Gaa & $\rightarrow$ & Taa & & Glu & $\rightarrow$ & Stop & & $2^{a}$ & 1 & & $3^{b}$ & \\
\hline & 409 & Cag & $\rightarrow$ & Aag & & Gln & $\rightarrow$ & Lys & 1 & & & & & \\
\hline & 413 & $\mathrm{cCg}$ & $\rightarrow$ & $\mathrm{cAg}$ & CpG & Pro & $\rightarrow$ & Gln & & 1 & & 1 & & \\
\hline $\mathrm{G}: \mathrm{C} \rightarrow \mathrm{C}: \mathrm{G}$ & 6 & $\mathrm{agC}$ & $\rightarrow$ & agG & CpG & Ser & $\rightarrow$ & Arg & & 1 & & & & \\
\hline & 50 & $c G t$ & $\rightarrow$ & $c C t$ & CpG & Arg & $\rightarrow$ & Pro & & & & 1 & & \\
\hline & 110 & cGt & $\rightarrow$ & $\mathrm{cCt}$ & CpG & Arg & $\rightarrow$ & Pro & & & & & 1 & \\
\hline & 112 & $\mathrm{Ggc}$ & $\rightarrow$ & $\mathrm{Cgc}$ & & Gly & $\rightarrow$ & Arg & 1 & & & & 1 & \\
\hline & 125 & $\mathrm{cCg}$ & $\rightarrow$ & $c G g$ & CpG & Pro & $\rightarrow$ & Arg & & 1 & & $2^{a}$ & & \\
\hline & 127 & Ggt & $\rightarrow$ & Cgt & & Gly & $\rightarrow$ & Arg & & & & & & 1 \\
\hline & 130 & Gcg & $\rightarrow$ & $\mathrm{Ccg}$ & & Ala & $\rightarrow$ & Pro & & & & & 1 & 1 \\
\hline & 139 & Gcg & $\rightarrow$ & $\mathrm{Ccg}$ & & Ala & $\rightarrow$ & Pro & & & & & 1 & \\
\hline & 186 & $\operatorname{agC}$ & $\rightarrow$ & $\mathrm{agG}$ & & Ser & $\rightarrow$ & Arg & 2 & 1 & & 1 & & \\
\hline & 206 & cGc & $\rightarrow$ & $\mathrm{cCc}$ & CpG & Arg & $\rightarrow$ & Pro & & & & & 1 & \\
\hline & 280 & Ggt & $\rightarrow$ & Cgt & CpG & Gly & $\rightarrow$ & Arg & & & 1 & 1 & & \\
\hline & 289 & Gcg & $\rightarrow$ & $\mathrm{Ccg}$ & & Ala & $\rightarrow$ & Pro & & & & 1 & & \\
\hline & 290 & $\mathrm{gCg}$ & $\rightarrow$ & $g G g$ & CpG & Ala & $\rightarrow$ & Gly & 1 & & & & & \\
\hline & 340 & Gca & $\rightarrow$ & $\mathrm{Cca}$ & CpG & Ala & $\rightarrow$ & Pro & 1 & 1 & 1 & 1 & $4^{b}$ & 1 \\
\hline & 413 & $\mathrm{cCg}$ & $\rightarrow$ & $c G g$ & CpG & Pro & $\rightarrow$ & Arg & & & & & 1 & \\
\hline & 418 & Gat & $\rightarrow$ & Cat & & Asp & $\rightarrow$ & $\mathrm{His}$ & & & & & 1 & \\
\hline & 442 & $\mathrm{Cca}$ & $\rightarrow$ & Gca & & Pro & $\rightarrow$ & Ala & 1 & & & & 1 & \\
\hline $\mathrm{A}: \mathrm{T} \rightarrow \mathrm{T}: \mathrm{A}$ & 10 & Aaa & $\rightarrow$ & Taa & & Lys & $\rightarrow$ & Stop & & 1 & & & & \\
\hline & 25 & Tgg & $\rightarrow$ & Agg & & Trp & $\rightarrow$ & Arg & & & & & $2^{\mathrm{a}}$ & 1 \\
\hline & 44 & $\mathrm{caT}$ & $\rightarrow$ & $\mathrm{caA}$ & & His & $\rightarrow$ & Gln & & & & & 1 & \\
\hline & 88 & Aaa & $\rightarrow$ & Taa & & Lys & $\rightarrow$ & Stop & & & & & & 2 \\
\hline & 146 & gAa & $\rightarrow$ & gTa & & Glu & $\rightarrow$ & Val & & $3^{a}$ & & & & \\
\hline & 164 & gTc & $\rightarrow$ & gAc & & Val & $\rightarrow$ & Asp & & & 2 & & & \\
\hline & 187 & Tac & $\rightarrow$ & $\mathrm{Aac}$ & & Tyr & $\rightarrow$ & Asn & 1 & & & & & \\
\hline & 365 & $\mathrm{gTt}$ & $\rightarrow$ & gAt & & Val & $\rightarrow$ & Asp & & & & & 1 & \\
\hline
\end{tabular}


Table 3 DNA sequence analysis of gpt mutations obtained from the lung of B[a]P-instilled and control mice (Continued)

\begin{tabular}{|c|c|c|c|c|c|c|c|c|c|c|c|c|c|}
\hline & 375 & taT & $\rightarrow$ & taA & Tyr & $\rightarrow$ & Stop & & 1 & & & & \\
\hline & 419 & gAt & $\rightarrow$ & gTt & Asp & $\rightarrow$ & Val & & & $2^{a}$ & & & \\
\hline & 458 & $\mathrm{tAa}$ & $\rightarrow$ & tTa & Stop & $\rightarrow$ & Leu & & 1 & & & & \\
\hline & 459 & taA & $\rightarrow$ & taT & Stop & $\rightarrow$ & Tyr & & & 1 & & & \\
\hline \multirow[t]{6}{*}{$A: T \rightarrow C: G$} & 1 & Atg & $\rightarrow$ & Ctg & Met & $\rightarrow$ & Leu & & & 1 & & & \\
\hline & 56 & cTc & $\rightarrow$ & cGc & Leu & $\rightarrow$ & $\operatorname{Arg}$ & & & 1 & & & 1 \\
\hline & 106 & Agc & $\rightarrow$ & $\mathrm{Cgc}$ & Ser & $\rightarrow$ & $\operatorname{Arg}$ & & 1 & 1 & & & \\
\hline & 134 & $\mathrm{tTa}$ & $\rightarrow$ & $\mathrm{tGa}$ & Leu & $\rightarrow$ & Stop & & 1 & & & & \\
\hline & 312 & tat & $\rightarrow$ & $\mathrm{taG}$ & Tyr & $\rightarrow$ & Stop & & & & & & 1 \\
\hline & 419 & gAt & $\rightarrow$ & $\mathrm{gCt}$ & Asp & $\rightarrow$ & Ala & & 1 & & & & 1 \\
\hline Deletion & $8-12$ & \multicolumn{3}{|c|}{ gAAAAAt } & & $\rightarrow$ & gAAAAt & 1 & 1 & 1 & & & \\
\hline \multirow[t]{17}{*}{-1 base } & 13 & \multicolumn{4}{|l|}{ aTa } & $\rightarrow$ & aа & & 1 & & & & \\
\hline & $26-28$ & \multicolumn{4}{|c|}{ tGGGa } & $\rightarrow$ & $\mathrm{tGGa}$ & & & & & 1 & 1 \\
\hline & $34-35$ & \multicolumn{4}{|c|}{$g \pi g$} & $\rightarrow$ & gTg & 1 & & & & & \\
\hline & 67 & \multicolumn{4}{|l|}{$\mathrm{aCt}$} & $\rightarrow$ & at & & & & & 1 & \\
\hline & $86-87$ & \multicolumn{4}{|c|}{$\mathrm{tGGa}$} & $\rightarrow$ & $\mathrm{tGa}$ & & & & & 1 & \\
\hline & $101-102$ & \multicolumn{4}{|c|}{ gCCg } & $\rightarrow$ & $\mathrm{gCg}$ & & & & & 1 & \\
\hline & 114 & \multicolumn{4}{|l|}{$\mathrm{gCg}$} & $\rightarrow$ & gg & & & 1 & & & \\
\hline & $124-125$ & \multicolumn{4}{|c|}{$\mathrm{aCCg}$} & $\rightarrow$ & $\mathrm{aCg}$ & & & & & & 1 \\
\hline & $126-128$ & \multicolumn{4}{|c|}{ cGGGt } & $\rightarrow$ & cGGt & & 1 & & & 1 & \\
\hline & 129 & \multicolumn{4}{|l|}{ gTg } & $\rightarrow$ & gg & & 1 & & & & \\
\hline & $170-171$ & \multicolumn{4}{|c|}{$\mathrm{aCCg}$} & $\rightarrow$ & $\mathrm{aCg}$ & & & 1 & & $2^{a}$ & \\
\hline & 176 & \multicolumn{4}{|l|}{$\mathrm{tGt}$} & $\rightarrow$ & $\mathrm{tt}$ & & & & & & 1 \\
\hline & 198 & \multicolumn{4}{|l|}{$\mathrm{aCa}$} & $\rightarrow$ & aа & & & & 1 & & \\
\hline & 217 & \multicolumn{4}{|l|}{$\mathrm{aGt}$} & $\rightarrow$ & at & & & & & 1 & \\
\hline & 244 & \multicolumn{4}{|l|}{ cGa } & $\rightarrow$ & $\mathrm{ca}$ & & & & 1 & & \\
\hline & $247-248$ & \multicolumn{4}{|c|}{$\mathrm{aGGc}$} & $\rightarrow$ & $a G c$ & & & & & & 1 \\
\hline & 249 & \multicolumn{4}{|l|}{$\mathrm{gCt}$} & $\rightarrow$ & gt & & & 1 & & & \\
\hline - & 266 & $g A c$ & & & & $\rightarrow$ & gc & & & 1 & & & \\
\hline & $270-271$ & $\mathrm{tGGt}$ & & & & $\rightarrow$ & $\mathrm{tGt}$ & & $2^{\mathrm{a}}$ & & 1 & & \\
\hline & $278-279$ & $\mathrm{aCCg}$ & & & & $\rightarrow$ & $\mathrm{aCg}$ & & & & & 1 & \\
\hline & 285 & gTa & & & & $\rightarrow$ & ga & & & & & 1 & \\
\hline & 293-294 & gTTg & & & & $\rightarrow$ & gTg & & & & & 1 & \\
\hline & $315-318$ & CAAA & & & & $\rightarrow$ & cAAAg & & & & & 1 & \\
\hline & 319 & aGc & & & & $\rightarrow$ & $\mathrm{ac}$ & & 1 & & & 1 & \\
\hline & $332-333$ & $\mathrm{aCCa}$ & & & & $\rightarrow$ & $\mathrm{aCa}$ & & 1 & & & & \\
\hline & $401-402$ & $\mathrm{tGGa}$ & & & & $\rightarrow$ & $\mathrm{tGa}$ & & & 1 & & & \\
\hline & $416-418$ & $\mathrm{tGGG}$ & & & & $\rightarrow$ & $\mathrm{tGGa}$ & 1 & 1 & & & 1 & \\
\hline & $442-443$ & gCCa & & & & $\rightarrow$ & gCa & & & & 1 & & \\
\hline$>2$ & 107-109 & $\mathrm{aGCC}$ & & & & $\rightarrow$ & ag & & & & & 2 & \\
\hline & $114-120$ & gCGC & Gg & & & $\rightarrow$ & gg & & & & & & 1 \\
\hline & 129-139 & gTGC & ACT & & & $\rightarrow$ & gc & & & 2 & & & \\
\hline & $140-152$ & gCGC & $\mathrm{BAAC}$ & GGt & & $\rightarrow$ & gt & & 1 & & & & \\
\hline & $161-442$ & & & & & & & & 1 & & & & \\
\hline
\end{tabular}


Table 3 DNA sequence analysis of gpt mutations obtained from the lung of B[a]P-instilled and control mice (Continued)

\begin{tabular}{|c|c|c|c|c|c|c|c|c|c|c|}
\hline & $243-248$ & gCGAAGGc & $\rightarrow$ & gc & & & & & & 1 \\
\hline & 300-306 & tTCGTGAAa & $\rightarrow$ & ta & & & & & & 1 \\
\hline & $375-380$ & aTGTTGTt & $\rightarrow$ & at & & & 2 & & & \\
\hline \multirow[t]{12}{*}{ Insertion } & 25 & $\mathrm{cTg}$ & $\rightarrow$ & $\mathrm{cTTg}$ & & & & & 1 & \\
\hline & 75 & $\mathrm{ct}$ & $\rightarrow$ & cAt & & $3^{a}$ & & & & \\
\hline & 124 & ac & $\rightarrow$ & aTc & & & & & $2^{a}$ & \\
\hline & 136 & $\mathrm{aCt}$ & $\rightarrow$ & $\mathrm{aCCt}$ & & & & & 1 & \\
\hline & $223-225$ & gAAAc & $\rightarrow$ & gAAAAc & & 1 & & & & \\
\hline & 229 & cGc & $\rightarrow$ & cGGc & & & & & & 1 \\
\hline & 286 & gt & $\rightarrow$ & gATACCGGTGGt & & 1 & & & & \\
\hline & $335-337$ & $\mathrm{aTCTt}$ & $\rightarrow$ & aTCTTCTt & & & & & & 1 \\
\hline & 362 & cTg & $\rightarrow$ & $\mathrm{cTTg}$ & & & & & 1 & \\
\hline & 390 & $\mathrm{CC}$ & $\rightarrow$ & cTc & & & & & 1 & \\
\hline & 392-393 & CAAg & $\rightarrow$ & CAAAg & & & & & & 1 \\
\hline & $401-402$ & $\mathrm{tGGa}$ & $\rightarrow$ & tGGGa & & & 1 & 2 & & \\
\hline \multirow[t]{5}{*}{ Other } & $26-27$ & $\mathrm{tGGg}$ & $\rightarrow$ & $\mathrm{tTg}$ & & & & 1 & & \\
\hline & $59-60$ & gCAa & $\rightarrow$ & gGa & & & & & 1 & \\
\hline & 100-102 & $\mathrm{tGCCg}$ & $\rightarrow$ & $\mathrm{tTg}$ & & & & & 1 & \\
\hline & $140-141$ & gCGC & $\rightarrow$ & gAAC & & & & 1 & & \\
\hline & 304 & $\mathrm{tGa}$ & $\rightarrow$ & tAAa & & 1 & & & & \\
\hline Total & & & & & 38 & 92 & 43 & 42 & 79 & 49 \\
\hline
\end{tabular}

methylated, by spontaneous deamination of methylated cytosines to form thymine residues, resulting in the formation of G:T mispairs [25]. Most cytosines in CpG sites in the liver of gpt gene integrated in the genomic DNA are highly methylated [26], and are therefore hotspots of spontaneous mutation in the control mice, such as G:C to A:T transitions at nucleotides 64,110 , and 115 are at $\mathrm{CpG}$ sites (Table 3). Methyl-CpG Domain Protein 4 (MBD4) and thymine-DNA glycosylase (TDG) [27] are mismatch repair enzymes that correct G:T mispairs by excising the mispaired thymine [28]. We consider that decrease in these DNA glycosylases and other mismatch repair enzymes possibly contribute to the observed increase in occurrence of $\mathrm{G}: \mathrm{C}$ to $\mathrm{A}: \mathrm{T}$ transitions in aged animals [19, 21, 29].

In $\mathrm{B}[\mathrm{a}] \mathrm{P}$-instilled lung of $g p t$ delta mice, $\mathrm{G}: \mathrm{C}$ to $\mathrm{A}: \mathrm{T}$ transitions were shown to increase in an age-dependent manner (Table 2); this type of mutation was most predominant in 24-month-old mice. In contrast, G:C to $\mathrm{T}$ :A transversions (a landmark mutation of $\mathrm{B}[\mathrm{a}] \mathrm{P}-\mathrm{DNA}$ adduct formation possibly induced by translesional DNA synthesis [30]) were the major base substitution in $\mathrm{B}$ [a]P-instilled lungs of 3-month-old mice. As summarized in Table 4, estimation of specific mutant frequency ([Average mutant frequency in Table 1] $\times[\%$ mutant of $\mathrm{G}: \mathrm{C}$ to $\mathrm{A}: \mathrm{T}$ transition or $\mathrm{G}: \mathrm{C}$ to $\mathrm{T}: \mathrm{A}$ transversion of

Table 4 Specific mutant frequency of G:C to A:T transition and G:C to T:A transversion on gpt gene from the lung of B[a]P-instilled and control mice

\begin{tabular}{|c|c|c|c|c|c|c|}
\hline \multirow[t]{2}{*}{ Type of mutation in the gpt gene } & \multicolumn{3}{|c|}{ Control (months) } & \multicolumn{3}{|c|}{$\mathrm{B}[\mathrm{a}] \mathrm{P}$ (months) } \\
\hline & 3 & 11 & 24 & 3 & 11 & 24 \\
\hline & \multicolumn{6}{|c|}{ Specific mutant frequency* $\left(\times 10^{-5}\right)$} \\
\hline $\mathrm{G}: \mathrm{C} \rightarrow \mathrm{A}: \mathrm{T}$ & 0.27 & 0.44 & 0.35 & 0.25 & 0.42 & $0.92^{* *}$ \\
\hline $\mathrm{G}: \mathrm{C} \rightarrow \mathrm{T}: \mathrm{A}$ & 0.12 & 0.18 & 0.14 & 1.39 & 0.40 & 0.41 \\
\hline
\end{tabular}

${ }^{*}$ Specific mutant frequency $=$ [Average mutant frequency in Table 1] $\times[\%$ mutant of G:C to A:T transition or G:C to T:A transversion of corresponding group in Table 2]

**P $P 0.001$ (Chi-square test) for comparison between $\mathrm{B}[a] \mathrm{P}$-instilled mice and age-matched control mice 
corresponding group in Table 2]) showed that G:C to T:A transversion was markedly increased in $\mathrm{B}[\mathrm{a}] \mathrm{P}$-instilled lung of 3-month-old mice $\left(1.39 \times 10^{-5}\right)$ compared to the age-matched control $\left(0.12 \times 10^{-5}\right)$ but the increase of $\mathrm{G}: \mathrm{C}$ to A:T transition by $\mathrm{B}[\mathrm{a}] \mathrm{P}$ instillation was not observed in 3-month-old mice. On the other hand, in B[a]Pinstilled lung of 24-month-old mice, specific mutant frequency of G:C to A:T transition $\left(0.92 \times 10^{-5}\right)$ was elevated significantly $(P<0.01$, Chi-square test $)$ compared to the age-matched control $\left(0.35 \times 10^{-5}\right)$, while $\mathrm{G}: \mathrm{C}$ to $\mathrm{T}: \mathrm{A}$ transversion was also elevated by $\mathrm{B}[\mathrm{a}] \mathrm{P}$ instillation. These observations suggest that $\mathrm{G}: \mathrm{C}$ to $\mathrm{T}: \mathrm{A}$ transversion was a predominant mutation for elevation of mutant frequency in $\mathrm{B}$ [a]P-instilled lung of 3-month-old mice, but in 24-month-old mice, induction of G:C to A:T transition as well as $\mathrm{G}: \mathrm{C}$ to $\mathrm{T}: \mathrm{A}$ transversion drove the elevation of mutant frequency by $\mathrm{B}[\mathrm{a}] \mathrm{P}$ instillation. Elevated levels of metabolic activation in young animals [22] might accelerate the induction of $\mathrm{G}: \mathrm{C}$ to $\mathrm{T}: \mathrm{A}$ transversions.

Our mutation spectrum analysis of B[a]P-instilled lungs revealed that not only the overall percentage of $\mathrm{G}: \mathrm{C}$ to A:T transitions but the percentage of $\mathrm{G}: \mathrm{C}$ to $\mathrm{A}: \mathrm{T}$ transitions at non-CpG sites increased with age $(8 \%, 12 \%$, and $25 \%$ ['the percentage of total G:C to A:T transitions' minus 'the percentage of total $\mathrm{G}: \mathrm{C}$ to $\mathrm{A}: \mathrm{T}$ transitions at CpG sites'] at 3 months, 11 months, and 24 months, respectively). A possible mechanism for the induction of $\mathrm{G}: \mathrm{C}$ to A:T transitions in $\mathrm{B}[\mathrm{a}] \mathrm{P}$-instilled old mice is that spontaneous deamination of cytosine at $\mathrm{CpG}$ sites was elevated in the lungs of these mice by instillation of $\mathrm{B}[\mathrm{a}] \mathrm{P}$, resulting in an increase in the percentage of $\mathrm{G}: \mathrm{C}$ to $\mathrm{A}: \mathrm{T}$ transitions at CpG sites in 24-month-old mice (16\%). A decrease in mismatch repair [29] might also contribute to the increase in occurrence of $\mathrm{G}: \mathrm{C}$ to $\mathrm{A}: \mathrm{T}$ transitions in $\mathrm{B}[\mathrm{a}] \mathrm{P}$-instilled aged animals. Another possibility is that DNA cytidine deaminase is activated in the lungs of aged mice by instillation of $\mathrm{B}[\mathrm{a}] \mathrm{P}$. This enzyme catalyzes the conversion of cytosine to uracil at both $\mathrm{CpG}$ and nonCpG sites, which leads to G:U mispair formation and hence mutation of G:C to A:T. The hypermutation induced by DNA cytidine deaminase plays a role in creating antibody diversity in the variable regions [31], and expression of this enzyme causes genomic instability related to cancer and other diseases [32]. We speculate that $\mathrm{B}[\mathrm{a}] \mathrm{P}$ induces DNA cytidine deaminase in the lungs of aged animals resulting in the induction of $\mathrm{G}: \mathrm{C}$ to $\mathrm{A}: \mathrm{T}$ transversions at non-CpG sites.

The biological significance of the age-related increase in G:C to A:T transitions in both B[a]P-instilled and control mice remains unclear. G:C to A:T transitions are induced on codons 12 and 13 of the K-ras gene in lung tumors spontaneously induced in either p53-suppressed old mice or age-matched wild-type mice (13- to 24-month-old mice) [33]. Recently, G:C to A:T transitions were shown to occur frequently in six types of human tumors (lung adenocarcinoma, lung squamous cell carcinoma], bladder, cervix, head and neck), in which APOBEC3B, an isoform of DNA-cytidine deaminase, was upregulated [34], and lung adenocarcinoma developed in transgenic mice with constitutive expression of DNA-cytidine deaminase [35]. These observations suggest that an increase in $\mathrm{G}: \mathrm{C}$ to A:T transitions may contribute to not only spontaneous but also $\mathrm{B}[\mathrm{a}] \mathrm{P}$-induced tumorigenesis in the lungs of aged mice. However, further studies are required to reveal the mechanism that G:C to A:T transitions induced in the lung cause cancer and other diseases in the old age.

\section{Conclusions}

Our observations indicate that the order of the age groups in terms of highest to lowest fold of increase in mutant frequency in the lungs following instillation of $\mathrm{B}[\mathrm{a}] \mathrm{P}$ was 3-, 24-, and 11-month-old mice, suggesting that young mice are the age-group most susceptible to $\mathrm{B}[\mathrm{a}] \mathrm{P}$. G:C to T:A transversion was shown to be a predominant mutation for elevation of mutant frequency in B[a]P-instilled lung of 3month-old mice, but in 24-month-old mice, induction of G:C to A:T transition as well as G:C to T:A transversion drove the elevation of mutant frequency by $\mathrm{B}[\mathrm{a}] \mathrm{P}$ instillation. We speculate that $\mathrm{B}[\mathrm{a}] \mathrm{P}$ induces DNA cytidine deaminase in the lungs of aged animals resulting in the induction of G:C to A:T transversions at non-CpG sites.

\section{Competing interests}

The authors declare that they have no competing interests.

\section{Authors' contributions}

YA conceived of the study, and participated in its design and coordination. $\mathrm{AHH}$ participated in the design of study, and performed the experiments and statistical analysis in this study. YS performed the experiments and statistical analysis in this study. KHA carried out intratracheal administration. SG participated in the design of study. KM and TN developed gpt delta mice and the mutation assay system, and helped to carry out the mutation assay in NIES. All authors read and approved the final manuscript.

\section{Acknowledgements}

We thank Dr. Takehiro Michikawa (NIES) for his helpful suggestion for statistical analysis. This work was partly supported by a Grant-in-Aid of the Japan Science Promotion Society (\#17390037 to YA, SG and TN).

\section{Author details}

${ }^{1}$ National Institute for Environmental Studies, Center for Environmental Risk Research, 16-2 Onogawa, 305-8506 Tsukuba, Ibaraki, Japan. Juntendo University, Graduate School of Health and Sports Science, 270-1695 Inzai, Chiba, Japan. 'Division of Genetics and Mutagenesis, National Institute of Health Sciences, 158-8501 Setagaya-ku, Tokyo, Japan. ${ }^{4}$ Present address: University of Shizuoka, School of Nursing, 422-8526 Suruga-ku, Shizuoka, Japan.

Received: 19 August 2014 Accepted: 3 March 2015

Published online: 16 June 2015

\section{References}

1. Vijg J, Suh Y. Genome instability and aging. Ann Rev Physiol. 2013;75:645-68.

2. Koyama N, Yasui M, Kimura A, Takami S, Suzuki T, Masumura K, et al. Acrylamide genotoxicity in young versus adult gpt delta male rats. Mutagenesis. 2011;26:545-9. 
3. Lambert IB, Singer TM, Boucher SE, Douglas GR. Detailed review of transgenic rodent mutation assays. Mutat Res. 2005;590:1-280.

4. Gossen JA, de Leeuw WJ, Vijg J. LacZ transgenic mouse models: their application in genetic toxicology. Mutat Res. 1994;307:451-9.

5. Martus HJ, Dolle ME, Gossen JA, Boerrigter ME, Vijg J. Use of transgenic mouse models for studying somatic mutations in aging. Mutat Res. 1995;338:203-13.

6. Vijg J, Dolle ME, Martus HJ, Boerrigter ME. Transgenic mouse models for studying mutations in vivo: applications in aging research. Mech Ageing Develop. 1997:99:257-71

7. Ono T, Ikehata H, Pithani VP, Uehara Y, Chen Y, Kinouchi Y, et al. Spontaneous mutations in digestive tract of old mice show tissue-specific patterns of genomic instability. Cancer Res. 2004;64:6919-23.

8. Hill KA, Buettner VL, Halangoda A, Kunishige M, Moore SR, Longmate J, et al. Spontaneous mutation in Big Blue mice from fetus to old age: tissue-specific time courses of mutation frequency but similar mutation types. Environ Mol Mutag. 2004;43:110-20.

9. Hill KA, Halangoda A, Heinmoeller PW, Gonzalez K, Chitaphan C, Longmate J, et al. Tissue-specific time courses of spontaneous mutation frequency and deviations in mutation pattern are observed in middle to late adulthood in Big Blue mice. Environ Mol Mutag. 2005;45:442-54.

10. Li W, Vijg J. Measuring genome instability in aging - a mini-review. Gerontology. 2012;58:129-38.

11. Hashimoto AH, Amanuma K, Hiyoshi K, Takano H, Masumura K, Nohmi T, et al. In vivo mutagenesis induced by benzo[a]pyrene instilled into the lung of gpt delta transgenic mice. Environ Mol Mutag. 2005;45:365-73.

12. Nohmi T, Katoh M, Suzuki H, Matsui M, Yamada M, Watanabe M, et al. A new transgenic mouse mutagenesis test system using Spi- and 6-thioguanine selections. Environ Mol Mutag. 1996;28:465-70.

13. Takano H, Yanagisawa R, Ichinose T, Sadakane K, Inoue K, Yoshida S, et al. Lung expression of cytochrome P450 1A1 as a possible biomarker of exposure to diesel exhaust particles. Arch Toxicol. 2002;76:146-51.

14. Nohmi T, Suzuki T, Masumura K. Recent advances in the protocols of transgenic mouse mutation assays. Mutat Res. 2000;455:191-215.

15. Adams WT, Skopek TR. Statistical test for the comparison of samples from mutational spectra. J Mol Biol. 1987;194:391-6.

16. Cariello NF, Piegorsch WW, Adams WT, Skopek TR. Computer program for the analysis of mutational spectra: application to p53 mutations. Carcinogenesis. 1994;15:2281-5.

17. Hashimoto AH, Amanuma K, Hiyoshi K, Takano H, Masumura K, Nohmi T, et al. In vivo mutagenesis in the lungs of gpt-delta transgenic mice treated intratracheally with 1,6-dinitropyrene. Environ Mol Mutag. 2006;47:277-83.

18. Hashimoto AH, Amanuma K, Hiyoshi K, Sugawara Y, Goto S, Yanagisawa R, et al. Mutations in the lungs of gpt delta transgenic mice following inhalation of diesel exhaust. Environ Mol Mutag. 2007;48:682-93.

19. Ono T, Ikehata H, Nakamura S, Saito $Y$, Hosoi $Y$, Takai $Y$, et al. Age-associated increase of spontaneous mutant frequency and molecular nature of mutation in newborn and old lacZ-transgenic mouse. Mutat Res. 2000:447:165-77.

20. Swiger RR, Cosentino L, Masumura KI, Nohmi T, Heddle JA. Further characterization and validation of gpt delta transgenic mice for quantifying somatic mutations in vivo. Environ Mol Mutag. 2001;37:297-303.

21. Iyama T, Wilson 3rd DM. DNA repair mechanisms in dividing and non-dividing cells. DNA Repair. 2013;12:620-36.

22. Warrington JS, Court MH, Greenblatt DJ, von Moltke LL. Phenacetin and chlorzoxazone biotransformation in aging male Fischer 344 rats. J Pharm Pharmacol. 2004:56:819-25.

23. Dolle ME, Snyder WK, Dunson DB, Vijg J. Mutational fingerprints of aging Nucl Acids Res. 2002;30:545-9.

24. Masumura K, Matsui K, Yamada M, Horiguchi M, Ishida K, Watanabe M, et al, Characterization of mutations induced by 2-amino-1-methyl-6-phenylimidazo $[4,5-b]$ pyridine in the colon of gpt delta transgenic mouse: novel $\mathrm{G}: \mathrm{C}$ deletions beside runs of identical bases. Carcinogenesis. 2000;21:2049-56.

25. Gonzalgo ML, Jones PA. Mutagenic and epigenetic effects of DNA methylation. Mutat Res. 1997;386:107-18.

26. Takumi S, Aoki Y, Sano T, Suzuki T, Nohmi T, Nohara K. In vivo mutagenicity of arsenite in the livers of gpt delta transgenic mice. Mutat Res. 2014;760:42-7.

27. Hendrich $\mathrm{B}$, Hardeland $\mathrm{U}, \mathrm{Ng} \mathrm{HH}$, Jiricny J, Bird A. The thymine glycosylase MBD4 can bind to the product of deamination at methylated CpG sites. Nature. 1999;401:301-4.
28. Sjolund AB, Senejani AG, Sweasy JB. MBD4 and TDG: multifaceted DNA glycosylases with ever expanding biological roles. Mutat Res. 2013;743-744:12-25

29. Hsieh P, Yamane K. DNA mismatch repair: molecular mechanism, cancer, and ageing. Mech Ageing Develop. 2008;129:391-407.

30. Moriya M, Spiegel S, Fernandes A, Amin S, Liu T, Geacintov N, et al. Fidelity of translesional synthesis past benzo [a] pyrene diol epoxide-2'-deoxyguanosine DNA adducts: marked effects of host cell, sequence context, and chirality. Biochemistry. 1996;35:16646-51.

31. Honjo T, Kinoshita K, Muramatsu M. Molecular mechanism of class switch recombination: linkage with somatic hypermutation. Ann Rev Immunol. 2002;20:165-96.

32. Pham P, Bransteitter R, Goodman MF. Reward versus risk: DNA cytidine deaminases triggering immunity and disease. Biochemistry. 2005:44:2703-15.

33. Duan W, Gao L, Wu X, Hade EM, Gao JX, Ding H, et al. Expression of a mutant p53 results in an age-related demographic shift in spontaneous lung tumor formation in transgenic mice. PLoS One. 2009:4:e5563.

34. Burns MB, Temiz NA, Harris RS. Evidence for APOBEC $3 B$ mutagenesis in multiple human cancers. Nature Genet. 2013;45:977-83.

35. Okazaki IM, Hiai H, Kakazu N, Yamada S, Muramatsu M, Kinoshita K, et al. Constitutive expression of AID leads to tumorigenesis. J Exp Med. 2003;197:1173-81.

\section{Submit your next manuscript to BioMed Central and take full advantage of:}

- Convenient online submission

- Thorough peer review

- No space constraints or color figure charges

- Immediate publication on acceptance

- Inclusion in PubMed, CAS, Scopus and Google Scholar

- Research which is freely available for redistribution 